\title{
Acute Liver Damage and Anorexia Nervosa: A Case Report and Review of the Literature
}

\author{
Lionel Bridet, Juan Jose Beitia Martin, Jose Luis Cabriada Nuno
}

\begin{abstract}
We treated a patient suffering from severe anorexia nervosa [body mass index (BMI), $10.5 \mathrm{~kg} / \mathrm{m}^{2}$ ] complicated with hepatic insufficiency. The treatment consisted of rehydration and the correction of her malnutrition, using adapted protocols to avoid the development of the refeeding syndrome. In view of her successful progress she was discharged from the hospital on day 50. Although we do not perform a hepatic biopsy, the mechanism seems to be related to autophagy more than hypoperfusion because of the extremely low BMI. This observation also highlights the need of following a multidisciplinary approach in these difficult cases $\left(\mathrm{BMI}<13 \mathrm{~kg} / \mathrm{m}^{2}\right)$, involving intensive care unit doctors, gastroenterologists, endocrinologists and psychiatrists.
\end{abstract}

Abbreviations: BMI: Body mass index; AST: Aspartate aminotransferase; ALT: Alanine aminotransferase; CT: Computed tomography.

Keywords: Anorexia nervosa, Liver damage, Multidisciplinary support.

How to cite this article: Bridet L, Martin JJB, Nuno JLC. Acute Liver Damage and Anorexia Nervosa: A Case Report and Review of the Literature. Euroasian J Hepato-Gastroenterol 2012;2(2):104-106.

\section{Source of support: Nil}

Conflict of interest: None

\section{INTRODUCTION}

Anorexia nervosa is an eating disorder characterized by voluntary weight loss, distortion of body image and intense fear of gaining weight. Several studies have described an increase in serum liver enzymes in severely malnourished patients who have been affected by anorexia nervosa. ${ }^{1,2}$ These are usually mild and normal values are restored when the body weight is normalized. The hepatic complications are usually related to an isolated elevation of the transaminase levels but, nevertheless, very few cases of acute liver failure in anorexia nervosa have been described. ${ }^{3-7}$ We are presenting the case of a 35-year-old woman who suffers from severe anorexia nervosa. She was admitted in June 2011 at our hospital following a hypoglycemic coma and acute liver damage.

\section{CASE REPORT}

Our patient, who works as a dietician, has been under psychiatric care since 1989 due to anorexia nervosa complicated with amenorrhea and osteoporosis. She was not prescribed any current regular medication although she admitted to using abusing laxatives (Bisacodyl) or diuretics. Further, she used to take occasionally half tablet of paracetamol for headaches. She does not consume alcohol regularly and denies any other toxic habits. Her last recorded weight was $32 \mathrm{~kg}$ in 2006. Her aminotransferase levels were normal [aspartate aminotransferase (AST); $2 \mathrm{U} / \mathrm{l}$ and alanine aminotransferase (ALT); $22 \mathrm{U} / \mathrm{l}]$.

The patient was seen initially at the emergency service in view of a decreased level of consciousness secondary to severe hypoglycemia and acute hepatic insufficiency. At the time of admission she presented with hypotension, 90/ $60 \mathrm{~mm} \mathrm{Hg}$; bradycardia, 46 beats per minute and her body temperature was $36^{\circ} \mathrm{C}$. The electrocardiogram showed sinus rhythm and a heart rate of 50 beats per minute. She had moderate diffuse and unspecific abdominal pain. There were no signs of hepatic encephalopathy. She was dramatically slim on admission. Her weight was $25 \mathrm{~kg}$ and her body mass index (BMI) was $10.5 \mathrm{~kg} / \mathrm{m}^{2}$. She reported a $3 \mathrm{~kg}$ weight loss within the previous weeks in the context of extreme anorexia.

Her full blood count showed leukopenia $\left(2,400 / \mathrm{mm}^{3}\right)$ and moderate thrombocytopenia $\left(65,000 / \mathrm{mm}^{3}\right)$. Regarding her blood biochemistry, there was marked hypoglycemia (16 mg/dl) and normal urea (80 mg/dl) and creatinine (0.58 $\mathrm{mg} / \mathrm{dl}$ ) levels. She did not have acidosis. Her $\mathrm{pH}$ was 7.36 and her bicarbonate level was normal $(40 \mathrm{mEq} / \mathrm{l})$. Her blood test also showed hypokalemia $(2.45 \mathrm{mEq} / \mathrm{l})$, hypocalcemia ( $7.3 \mathrm{mg} / \mathrm{dl})$, hypophosphatemia $(0.42 \mathrm{mg} / \mathrm{dl})$ and hypomagnesemia $(1.48 \mathrm{mg} / \mathrm{dl})$. Nutritionnaly, there was hypoproteinemia (4 gm/dl) and hypoalbuminemia (2.4 gm/dl).

On admission, she also had a marked elevation of transaminase levels: AST, 3,758 U/l (reference range, 1-40 U/l), ALT, 1,955 U/l (reference, 1-37 U/l). Gamma glutamyl transpeptidase levels were $280 \mathrm{U} / \mathrm{l}$ (7-32) and alkaline phosphatase was 344 U/l (30-106). Plasma bilirubin level was normal. Prothrombin time was 52\% and INR was 1.69 .

The serum paracetamol level was negative. The urinary toxicology screen (cannabis, cocaine, amphetamines, opiates, methadone, benzodiazepines) was negative.

Serologic tests for hepatotropic viruses (hepatitis A virus, hepatitis B virus, hepatitis C virus, herpes simplex virus, Epstein-Barr virus) were negative. Antinuclear, antimitochondrial and antismooth muscle antibodies were negative. Alpha 1 antitrypsin and ceruleoplasmin levels were normal. 
Her brain computed tomorgraphic (CT) scan was normal. Her abdominal ultrasound scan showed a homogeneous hepatomegaly with normal echostructure, moderate ascites and bilateral pleural effusion. There was no abnormality detected in her gallbladder and biliary ducts. The echocardiogram showed a nondilated left ventricle with a normal systolic function, a mild pericardial effusion. There was no cardiac valve dysfunction. Ejection fraction was 63\%.

Despite treating her hypoglycemia, her Glasgow coma score did not improve and she was admitted to the intensive care unit for assisted ventilation. After 5 days the patient was transferred to the endocrinology ward where she was given vitamin and ionic support, as well as enteral nutrition through a nasogastric tube. Her caloric input started at 750 $\mathrm{Kcal} /$ day for 1 week, it was then increased to 1,200 Kcal/ day for a further 2 weeks and finally, and after removing the nasogastric catheter, it was set at 1,800 Kcal/day orally. Oral nutrition was authorized from week 3 following resolution of her abdominal pain, which is usual in these patients. ${ }^{8}$ Biologically, there was a marked decrease in her transaminase levels after day 3 , achieving normal levels as of day 20. Her prothrombin time also normalized on day 3 . Her protein electrophoresis showed and increased of her total protein level at $6 \mathrm{gm} / \mathrm{dl}$ and a serum albumin of $3.2 \mathrm{gm} / \mathrm{dl}$ on day 45. Leukopenia and thrombocytopenia was normalized. Her pericardial effusion and ascites resolved as soon as her serum albumin returned to normal levels.

The patient was transferred to the psychiatric ward on day 20 for the management of her eating disorder, once she had been stabilized biologicaly and nutritionally. Her weight was $32 \mathrm{~kg}$ and her BMI 13.5 on day 45 after admission.

\section{DISCUSSION}

Anorexia nervosa is an eating disorder that develops quickly into a very important feeding restriction. The illness affects young women mainly, with a prevalence of up to $1 \%$ in teenagers. ${ }^{9}$ In a meta-analysis done in 2002, mortality reached 5\%, being suicides and lethal complications of malnutrition (cardiac mostly) the two more frequent causes. ${ }^{10}$ Many of the systemic complications of anorexia nervosa involve the gastrointestinal tract and include pancreatitis, ${ }^{11}$ gastroduodenal symptoms ${ }^{12,13}$ and particularly hepatic disorders. The presence of hypoglycemia is not usual within the context of this condition. In our patient's case it is explained due to an associated hepatic insufficiency. However, in the context of anorexia nervosa it is usually severe, recurrent and difficult to correct. ${ }^{14}$ It usually happens in young adults rather than adolescents (average age: 34 years). ${ }^{15,16}$ Cytopenia might be the result of the gelatine transformation of the bone marrow with hypocellularity, as described by
Nishio in $2003,{ }^{17}$ and it is usually corrected completely and rapidly by enteral nutrition.

Hypokalemia can be explained by the abuse of laxatives and diuretics. The anasarca is explained by hypoalbuminemia. Her clinical situation was not secondarily complicated by heart failure despite the presence of hypokaliemia, hypophosphoremia and hypomagnesemia, which could have caused severe arrhythmias due to a QT elongation, as has been described in previous studies. ${ }^{18,19}$

Her caloric input on the first week was set at $30 \mathrm{Kcal} /$ $\mathrm{kg} /$ day, with a view to avoid developing refeeding syndrome, which could have worsened her hepatic function even more or have caused cardiac complications. ${ }^{20}$

In our case, we cannot explain her liver failure as secondary to any toxic, drug intake, autoimmune or infectious agent. A cardiac dysfunction was eliminated by cardiac investigations.

We did not perform a hepatic biopsy, as in most of the previously described cases, due to the resolution of her liver function and clinical and psychological conditions. Although the mechanism of liver injury in anorexia nervosa remains unclear, it is believed that the marked elevation of the transaminase levels, along with hepatic insufficiency, is related to the presence of global dehydration, subsequent hypovolemia and hypotension, and thus hypoxemia and hepatic hypoperfusion. ${ }^{6,7}$ But this hypothesis has not been rigorously demonstrated and the precise reason has not been elucidated.

The most important series of hepatic biopsies performed in 12 patients that suffered from anorexia $\left(\mathrm{BMI}<13 \mathrm{~kg} / \mathrm{m}^{2}\right)$ and hepatic failure date from 2008: The results showed significant glycogenic depletion but also numerous autophagosomes responsible for autophagy. In only $25 \%$ of the cases signs of apoptosis were seen, without any described signs of cell necrosis. It is possible that this 'nutrition autophagy', a defence mechanism in the case of anorexia, was the cause for the hepatocyte death. The stress of the endoplasmic reticulocyte might have been the initial trigger. ${ }^{21}$ Similar histology data had been seen in a previous case report in 2006. ${ }^{22}$ This autophagy might play a double role: When the loss of weight starts occurring the liver anomalies are moderate and the autophagy can endure this state. After that, when malnutrition worsens and the BMI lowers, the transaminase peak level and subsequent liver failure become evident. During this time hepatocytes are formed of numerous autophagosomes, which explains cell death by autophagy. This kind of cell death could be the explanation for the contrast noticed with regards to the significant increase of the aminotransferases detected and the absence of liver necrosis and apoptosis showed in the histology results. Finally, it is likely that starvation-induced 
autophagy was the principal mechanism involved in liver cell damage in this population.

\section{REFERENCES}

1. Mickley D, Greenfeld D, Quinlan DM, Roloff P, Zwas F. Abnormal liver enzymes in outpatients with eating disorders. Int J Eat Disord 1996;20:325-29.

2. Jones SC, Pieri LF, Losowsky MS. Abnormalities of liver function in severe anorexia nervosa. Eur Eat Disord Rev 1999;7:28-36.

3. Furuta S, Ozawa Y, Maejima K, et al. Anorexia nervosa with severe liver dysfunction and subsequent critical complications. Intern Med 1999;38:575-79.

4. Sakada M, Tanaka A, Ohta D, et al. Severe steatosis resulted from anorexia nervosa leading to fatal hepatic failure. $\mathrm{J}$ Gastroenterol 2006;41:714-15.

5. Rivera-Nieves J, Kozaiwa K, Rees Parrish C, Iezzoni J, Berg CB. Marked transaminase elevation in anorexia nervosa. Dig Dis Sci 2000;45:1959-63.

6. De Caprio C, Alfonso A, Senatore I, et al. Severe acute liver damage in anorexia nervosa: Two cases reports. Nutrition 2006;22;572-75.

7. Di Pascoli L, Lion A, Milazzo D, Caregaro L. Acute liver damage in anorexia nervosa. Int J Eat Disord 2004;36:114-17.

8. Waldholtz BD, Andersen AE. Gastrointestinal symptoms in anorexia nervosa. Gastroenterology 1990;98:1415-19.

9. Treatment of patients with eating disorders (3rd ed). American Psychiatric Association. Am J Psychiatry 2006;162 (7 suppl): 4-54.

10. Steinhausen HC. The outcome of anorexia nervosa in the 20th century. Am J Psychiatric 2002;159:1284-93.

11. Cox KL, Cannon RA, Ament ME, Phillips HE, Schaffer CB. Biochemical and ultrasonics abnormalities of the pancreas in anorexia nervosa. Dig Dis Sci 1983;28:225-29.

12. Buchman AL, Ament ME, Weiner M, Kodner A, Mayer EA. Reversal of megaduodenum and duodenal dysmotility associated with improvement in nutritional status in anorexia nervosa. Dig Dis Sci 1994;39:433-40.

13. Tweed-Kent AM, Fagenholz PJ, Alam HB. Acute gastric dilatation in a patient with anorexia nervosa binge/purge subtype. J Emerg Trauma Shock 2010;3:403-05.
14. Inui A, Uemoto $M$, Ueno $\mathrm{N}$, Morita S, Baba S. Rebound hipoglycemia after intravenous glucose in anorexia patients. Lancet 1996;347:323-24.

15. Rich LM, Caine MR, Findling JW, et al. Hypoglycemic coma in anorexia nervosa. Case report and review of the literature. Arch Intern Med 1990;150:894-95.

16. Ratcliffe PJ, Bevan JS. Severe hypoglycemia and sudden death in anorexia nervosa. Psychol Med 1985;15:679-81.

17. Nishio S, Yamada K, Yamada H, et al. Severe neutropenia with gelatinous bone marrow transformation in anorexia nervosa: A case report. Int J Eat Dis 2003;33:360-63.

18. Miller KK, Grinspoon SK, Ciampa J, Hier J, Herzog D, Klibanski A. Medical findings in outpatients with anorexia nervosa. Arch Intern Med 2005;165:561-66.

19. Hall RC, Hoffman RS, Beresford TP, Wooley B, Tice L, Hall AK. Hypomagnesemia in patients with eating disorders. Psychosomatics 1988;29:264-72.

20. Cooke RA, Chabers JB. Anorexia nervosa and the heart. Br J Hosp Med 1995;54:313-17.

21. Rautou PE, Cazals-Hatem D, Moreau R, et al. A possible role of starvation-induced hepatocyte autophagy. Gastroenterology 2008;135:840-48.

22. Tajiri K, Shimizu Y, Tsuneyama K, Sugiyama T. A case report of oxidative stress in a patient with anorexia nervosa. Int J Eat Disord 2006;39:616-18.

\section{ABOUT THE AUTHORS}

\section{Lionel Bridet (Corresponding Author)}

Department of Gastroenterology, Hospital Galdakao-Usansolo, Barrio Labeaga, s/n 48960, Galdakao (Bizkaia), Spain, Phone:+34 645329468 e-mail: lionelbridet@hotmail.fr

\section{Juan Jose Beitia Martin}

Department of Endocrinology, Hospital Galdakao-Usansolo, Barrio Labeaga, Galdakao (Bizkaia), Spain

\section{Jose Luis Cabriada Nuno}

Department of Gastroenterology, Hospital Galdakao-Usansolo, Barrio Labeaga, Galdakao (Bizkaia), Spain 